Condition 2: Unstable modes with given values of $k_{\perp}$ and $k_{z}\left(k_{z} \neq 0\right)$ exist if and only if

$$
D(0)<k_{\perp}^{2}+k_{z}^{2}<D(\infty){ }^{1} .
$$

Modes with $k_{z}=0$ are stable ${ }^{1}$. For $k_{z}{ }^{2} \ll k_{\perp}^{2}$ this may be approximated by $D(0)<k_{\perp}^{2}<D(\infty)$. For $D(0) \leqq 0$ Condition 2 reduces to Condition 1 . With our test equilibria and perturbations, one obtains $D(0) \leqq 0$ at arbitrary densities for $T_{\mathrm{e} \|} \lesssim 4 W_{\mathrm{i} \perp}$ ( $u \ll 1$ assumed) or for $T_{\mathrm{e} \perp} \lesssim 4 W_{\mathrm{i} \perp}(N \gg 1, u \gg 1$ assumed). In the opposite case, $D(0)>0$, Condition 2 is satisfied only for densities in the range

$$
n_{\text {crit }}<n_{0}<n_{\max }
$$

\section{Temporäres Bleichen \\ in dotierten Alkalihalogenidkristallen mit einem Rubinlaser}

\section{J. Hingsammer, J. Schwarzmüller, G. Wolfram und G. Gehrer}

Physik-Department der Technischen Hochschule München

(Z. Naturforschg. 22 a, 282-284 [1967] ; eingegangen am 7. Januar 1967)

Das temporäre Bleichen ist eine geeignete Methode, die kurzzeitige Entstehung und Umwandlung von Farbzentren in Alkalihalogeniden unter dem Einfluß von Bleichlicht zu untersuchen (z. B. 1, 2). Man mißt hierbei die Änderung der optischen Dichte eines verfärbten Kristalls, während mit einer Bleichlichtquelle - in vorliegender Arbeit ein Rubinlaser ${ }^{3}$ - in eine Absorptionsbande des Kristalls eingestrahlt wird.

Mit der Anordnung der Abb. 1 wurden temporäre Bleichspektren von verfärbten $\mathrm{KJ}-, \mathrm{KJ}: \mathrm{Ba}$ - und $\mathrm{KCl}: \mathrm{Sr}$ Kristallen bei der Temperatur des flüssigen Stickstoffes

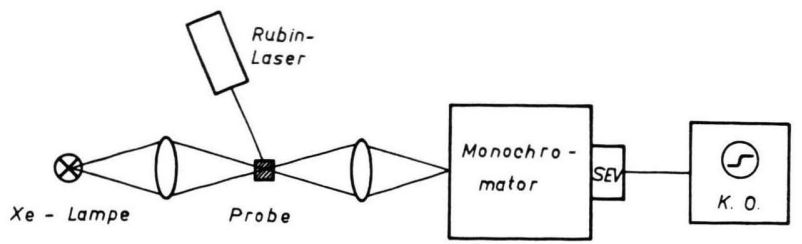

Abb. 1. Versuchsaufbau (schematisch).

aufgenommen, um daraus eine genauere Kenntnis der Elektronenkinetik im dotierten Alkalihalogenidkristall zu gewinnen. Beim KJ und KJ:Ba liegt die Laserlinie in der F-Bande, beim $\mathrm{KCl}: \mathrm{Sr}$ in der $\mathrm{Z}_{2}$-Bande. Der Vorteil der Untersuchung mit dem Laser liegt in der hohen Intensität des Laserlichts, die zu großen Änderungen der optischen Dichte führt.

1 J. Lambe u. W. D. Compton, Phys. Rev. 106, 694 [1957].

2 M. Ikezawa, M. Hirai u. M. Ueta, J. Phys. Soc. Japan 17, 1474 [1962].

3 K. РАRK, Phys. Rev. 140, A 1735 [1965].

4 H. PICK, Ann. Phys. 35, 73 [1939]. where $n_{\max }$ depends again on the magnetic field strength and other parameters. In our example, the density range for instability is given by

$$
\begin{aligned}
0<\frac{48 N}{\pi^{2}} F(N, 0,0,1)-\frac{W_{i \perp}}{T_{\mathrm{e} \perp}}\left[1-e^{-u} I_{0}(u)\right] \\
\quad-\frac{N^{2} \pi^{2}}{2}\left(\frac{\Omega_{\mathrm{i}}}{\omega_{\mathrm{pi}}}\right)^{2} \\
<\frac{48 W_{\mathrm{i} \perp}}{N \pi^{4} T_{\mathrm{i}}} F(N, 1,0,0)+\frac{W_{\mathrm{i} \perp}}{T_{\mathrm{e} \|}} e^{-u} I_{0}(u) .
\end{aligned}
$$

In the case $N=1, u \ll 1$, this simplifies to $0<0.041-\left(\frac{\Omega_{\mathrm{i}}}{\omega_{\mathrm{pi}}}\right)^{2}<0.019 \frac{W_{\mathrm{i} \perp}}{T_{\mathrm{i} \|}}+0.20 \frac{W_{\mathrm{i}} \perp}{T_{\mathrm{e} \|}}$.

A more detailed report will appear elsewhere.

\section{$Z$-Banden in $\mathrm{KJ}: B a$}

In $\mathrm{KJ}: \mathrm{Ba}$ wurden Banden gefunden, die nach unserem Wissen in der Literatur noch nicht beschrieben sind. Durch Versuche in Analogie zum ausführlich untersuchten $\mathrm{KCl}: \mathrm{Sr}$ (z. B. ${ }^{4-6}$ ) konnten diese Banden als Z-Banden nachgewiesen werden.

Die Dotierung im KJ:Ba betrug $4 \cdot 10^{-3}$ Mol.-Proz. Ba. Die Kristalle wurden bei $560{ }^{\circ} \mathrm{C}$ in 21 Torr Kaliumdampf additiv nach der van Doonsschen Methode ${ }^{7}$ verfärbt; durch Tempern bei $400{ }^{\circ} \mathrm{C}$ und Abschrecken in Benzol entstand eine reine F-Bande. Einstrahlen in die F-Bande bei Zimmertemperatur erzeugte bei $730 \mathrm{~m} \mu$ eine neue Bande, während die F-Bande ausgebleicht wurde. Die neue Bande ist bei Zimmertemperatur stabil und wird durch Bleichlicht nicht verändert. Aus der Übereinstimmung mit den Verhältnissen in $\mathrm{KCl}: \mathrm{Sr}$ kann auf eine $\mathrm{Z}_{1}$-Bande geschlossen werden.

Beim langsamen Abkühlen des Kristalls nach dem Verfärben treten bei $780 \mathrm{~m} \mu$ und bei $500 \mathrm{~m} \mu$ Banden auf, die als $\mathrm{Z}_{2^{-}}$und $\mathrm{S}$-Bande gedeutet werden können. Nach Ohкura ${ }^{8}$ wächst mit der $\mathrm{Z}_{2}$-Bande auf der kürzerwelligen Seite der F-Bande die sogen. S-Bande, die einem Übergang in einen höheren angeregten Zustand des $Z_{2}$-Zentrums zugeordnet wird.

Weiter wurde die $Z_{1}$-Bande erzeugt und diese dann durch Erwärmen im Dunkeln in eine $F$ - und eine $Z_{2}$ Bande umgebildet ${ }^{6}$.

Zwischen $\mathrm{F}$ - und $\mathrm{Z}_{2}$-Bande besteht ein thermisches Gleichgewicht, das sich bei Temperaturerniedrigung in Richtung der $Z_{2}$-Bande verschiebt.

Außerdem wurden $\mathrm{KJ}: \mathrm{Ba}-\mathrm{Kristalle}$, die nur die $\mathrm{F}$ Bandenabsorption zeigten, durch Licht der Wellenlänge $\lambda=728 \mathrm{~m} \mu$ bei $67{ }^{\circ} \mathrm{C}$ mit Hilfe eines Interferenzfilters gebleicht und so eine intensive $Z_{2}$-Bande erzeugt ${ }^{9}$. $Z_{2^{-}}$ und F-Bande überlappen sich sehr stark. Eine Zerlegung beider Banden ergibt für die $Z_{2}$-Bande eine Halbwertsbreite von $(0,38 \pm 0,05) \mathrm{eV}$.

5 P. Camagni, G. Chiarotti, F. G. Fumi u L. Giulotto, Phil. Mag. 45, 225 [1954].

${ }^{6}$ F. Sertz, Phys. Rev. 83, 134 [1951].

7 Z. van Doorn, Rev. Sci. Instrum. 32, 755 [1961].

8 H. Онкura, Phys. Rev. 136, A 446 [1964].

9 K. Kojima, J. Phys. Soc. Jap. 19, 868 [1964]. 

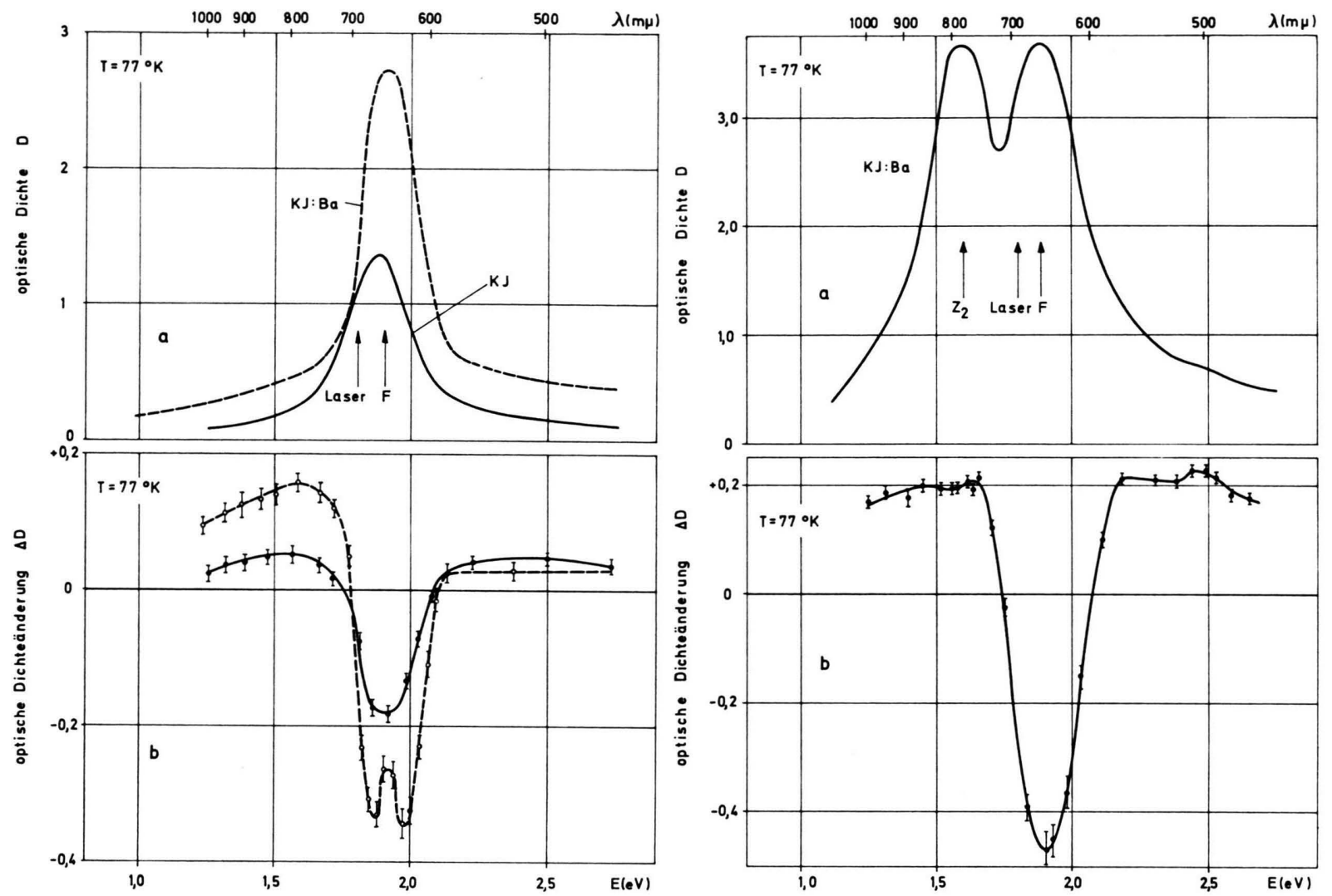

Abb. 2 a und b.

Abb. 3 a und $b$.

Abb. 2. a) F-Bandenabsorption in $\mathrm{KJ}$ und $\mathrm{KJ}: \mathrm{Ba}$; b) Bleichspektrum von $\mathrm{KJ}$ und $\mathrm{KJ}: \mathrm{Ba}$ beim Einstrahlen in die $\mathrm{F}$-Bande. Abb. 3. a) $\mathrm{F}$ - und $\mathrm{Z}_{2}$-Bande in $\mathrm{KJ}: \mathrm{Ba}$; b) Bleichspektrum von $\mathrm{KJ}: \mathrm{Ba}$ beim Einstrahlen in die F-Bande in Anwesenheit von $\mathrm{Z}_{\text {- }}$ Zentren.

Abb. 4. a) F- und $\mathrm{Z}_{2}$-Bande in $\mathrm{KCl}: \mathrm{Sr}$; b) Bleichspektrum beim Einstrahlen in die $\mathrm{Z}_{2}$-Bande.

\section{Bleichspektren}

Bei der Temperatur des flüssigen Stickstoffs wurde mit dem Rubinlaser in die F-Bande des $\mathrm{KJ}: \mathrm{Ba}$ und in die $\mathrm{Z}_{2}$-Bande des $\mathrm{KCl}: \mathrm{Sr}$ (molare $\mathrm{Sr}^{++}$-Konz. in der Schmelze $=10^{-3}$ ) eingestrahlt. Die beobachteten Bleichspektren folgen aus

$$
\Delta D(E)=-\log \left(1+\frac{\Delta I}{I}(E)\right)
$$

und sind in den Abb. 2-4 dargestellt ( $D=$ opt. Dichte). $\Delta I / I$ ist dabei die vom Laserlicht bewirkte relative Intensitätsänderung des transmittierten Meßlichts nach Ende des Laserpulses von etwa $400 \mu$ s Dauer.

Die Zerlegung der Bleichspektren ist im gegenwär- ${ }^{\circ}$ tigen Zeitpunkt ohne genaue Kenntnis der entstehen-o den Banden nicht möglich. An Hand der Abb. 2 kann jedoch festgestellt werden, daß unter der F-Bande eine: weitere Bande liegt. Die Abb. 4 läßt erkennen, daß mit dem Ausbleichen der $\mathrm{Z}_{2}$-Bande ein Ausbleichen beī̈ $440 \mathrm{~m} \mu$ und das Entstehen einer Bande bei $505 \mathrm{~m} \mu$ : verbunden ist. Aus Forn und Lage dieser Bande ${ }^{8}$ kann auf die $\mathrm{Z}_{3}$-Bande geschlossen werden, während es sich bei der Bande bei $440 \mathrm{~m} \mu$ um die von OhKura ${ }^{8}$ beschriebene S-Bande handeln dürfte, die auf einen

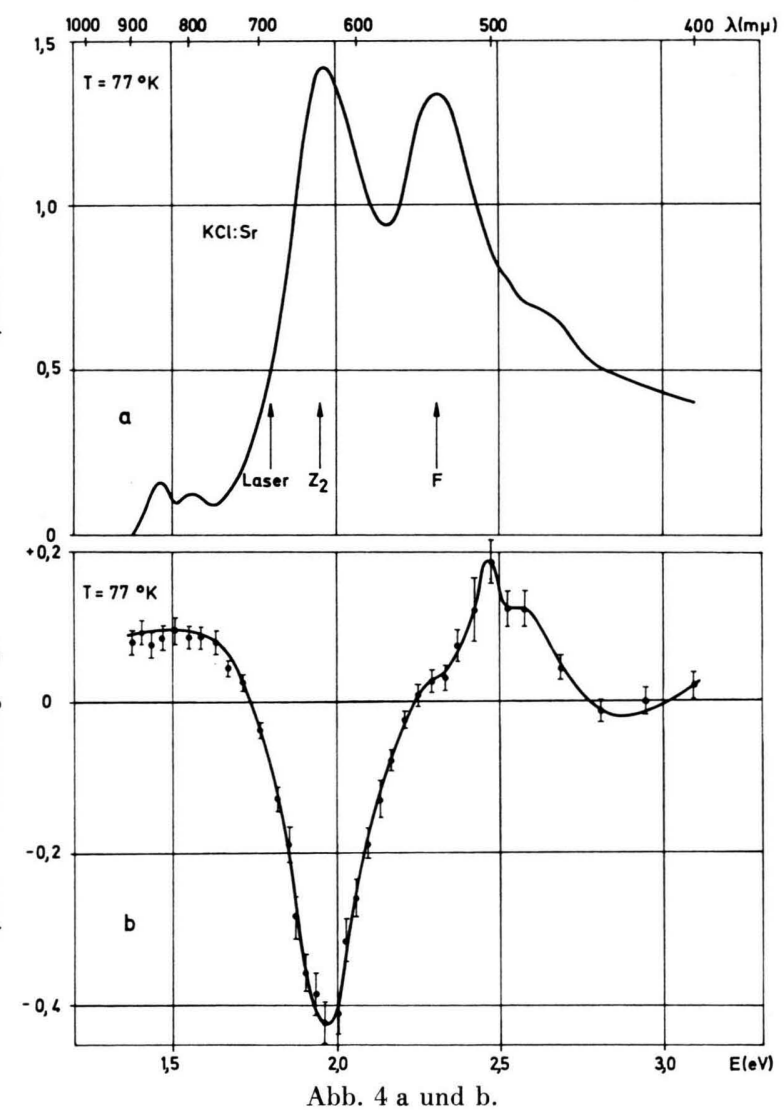


Übergang in ein höheres angeregtes Niveau zurückgeht. Das F-Zentrum scheint in den Umwandlungsprozeß $Z_{2} \rightarrow Z_{3}$ nicht verwickelt zu sein, da eine Änderung der F-Zentrenkonzentration im Bleichspektrum infolge der hohen Oszillatorstärke der F-Bandenabsorption deutlich sichtbar sein müßte.
Weitere Untersuchungen, auch mit EPR, sind im Gange.

Zu besonderem Dank sind wir Herrn Prof. Dr. E. Lüscher verpflichtet, der die Arbeit ermöglicht hat und stets förderte.

\section{Untersuchungen über InAs-Epitaxieschichten auf GaAs-Substraten}

\section{Günther Ernst Bauer}

Ludwig-Boltzmann-Institut für Festkörperphysik, Wien

(Z. Naturforschg. 22 a, 284-285 [1967]; eingeg. am 28. Dezember 1966)

Die elektronenoptische Untersuchung von epitaxial auf GaAs aufgewachsenen InAs-Schichten bestätigt die Regel von H. Holloway, nach der zur Elimination von Zwillingsbildung um so höhere Substrattemperaturen erforderlich sind, je kleiner der Winkel zwischen der Wachstums- und der jeweiligen $\langle 111\rangle$-Richtung ist.

a) Mit Hilfe einer chemischen Transportreaktion, bei der ein Gemisch von Wasserstoff und Wasserdampf als Trägergas diente, hatte RoBinson ${ }^{1}$ InAs-Schichten auf $\mathrm{GaAs}$ hergestellt, jedoch keine näheren Angaben über die Struktur der Schichten gemacht. Mit derselben Reaktion wurden von uns mit der sogen. Close Spaceoder Sandwich-Methode ${ }^{2-4}$ InAs-Schichten mit Schichtdicken zwischen $0,7 \mu$ und $2 \mu$ auf einkristallinen GaAsSubstraten der $\langle 100\rangle-,\langle 111\rangle$ - und $\langle 311\rangle$-Orientierungen aufwachsen gelassen. Die Substrattemperaturen lagen zwischen $700{ }^{\circ} \mathrm{C}$ und $775^{\circ} \mathrm{C}$. Die Schichten wurden mit Elektronenbeugung in Reflexionsstellung untersucht und zwar jeweils in einem $\langle 110\rangle$-Azimut.

b) Bei den auf $\mathrm{Ga}\{111\}$-Flächen aufgewachsenen Schichten konnte zunächst der Übergang zur azimutalen Orientierung beobachtet werden. Bei $750{ }^{\circ} \mathrm{C}$ Substrattemperatur zeigte sich dann ein Aufwachsen in doppelter Position: (111) InAs // (111) GaAs, $[0 \overline{1} 1]_{\mathrm{InAs}} / /[0 \overline{\mathrm{I}} 1]_{\mathrm{GaAs}}$ und $[01 \overline{1}]_{\text {InAs }} / /[0 \overline{1} 1]_{\mathrm{GaAs}}$. Diese Positionen treten mit gleicher Intensität auf, wie aus Abb. ${ }^{*}$ zu entnehmen ist; sie befinden sich zueinander in Zwillingsstellung nach der Oberflächennormale. Gleichzeitig sind auch noch die Zwillinge nach den zur Oberfläche geneigten $\langle 111\rangle$-Achsen, und zwar von beiden Aufwachspositionen vorhanden (in Abb. $1 T_{111}$ und $T_{111 / 111}$ ). Unter $750{ }^{\circ} \mathrm{C}$ Substrattemperatur zeigten sich auch zwei- und dreifache Zwillinge.

In den auf As\{111\}-Flächen aufgewachsenen Schichten zeigte sich, wie zu erwarten, ein höher geordnetes Wachstum, doch konnte auch bei $770{ }^{\circ} \mathrm{C}$ die Zwillingsbildung um die Oberflächennormale nicht verhindert werden.

1 P. H. Robinson, RCA Rev. 24, 574 [1963].

2 F. H. Nicold, J. Electrochem. Soc. 110, 1165 [1963].

3 E. Sirtl, J. Phys. Chem. Solids 24, 1285 [1963].

${ }^{4}$ H. J. Dersin u. E. Sirte, Z. Naturforschg. 21 a, 332 [1966].

* Abb. $1-3$ auf Tafel S. 284 a.
Bei $\{100\}$-Substraten zeigte sich bei $750{ }^{\circ} \mathrm{C}$ Substrattemperatur noch einfache Zwillingsbildung nach den vier zur Oberfläche geneigten $\langle 111\rangle$-Achsen. Diese Zwillinge verschwanden jedoch bei Erhöhung der Substrattemperatur und es trat paralleles Überwachsen auf (Abb. 2).

Auch auf den As $\{311\}$-Substraten konnte über $765{ }^{\circ} \mathrm{C}$ Substrattemperatur ein von Zwillingsbildung freies InAs-Wachstum erreicht werden.

Einige der hergestellten Schichten wurden schrittweise bis zur Zwischenfläche InAs-GaAs abgeätzt. Auf den Aufnahmen waren dann gemeinsam Reflexe von InAs und $\mathrm{GaAs}$ vorhanden, wie z. B. in Abb. 3 , in der auch schon die Zwillinge der InAs-Schicht nach [111] und $\lceil 1 \overline{1} \overline{1}]$ erkannt werden können (Substrattemperatur: $755^{\circ} \mathrm{C}$ ).

c) Es zeigte sich auch bei Schichten, die mit Substrattemperaturen um $700{ }^{\circ} \mathrm{C}$ hergestellt worden waren, kein Anzeichen für das Auftreten einer Wurtzitphase. Eine solche hatten Müller ${ }^{5}$, ebenso Holloway et al. ${ }^{6}$ beim $\mathrm{GaAs}$-Wachstum auf Ge festgestellt. Ein geordnetes hexagonales Aufwachsen, also mit den (0001)-Ebenen parallel zu den (111) des kubischen Gitters, führt zu Sechstelpunkten entlang den $\langle 111\rangle$-Geraden des reziproken Gitters. In unseren Aufnahmen konnten wir keine solchen feststellen. Die Debye-Ringe in Aufnahmen von Schichten, die bei tiefen Substrattemperaturen entstanden, wurden ausgemessen. Berücksichtigt man, daß Deвye-Ringe auch durch Doppelbeugung entstehen können, so verwendet man zum sicheren Nachweis einer hexagonalen Phase am besten den (10i1)Reflex ${ }^{7}$. Dieser Reflex konnte nicht beobachtet werden, es trat daher auch keine ungeordnete hexagonale Phase auf.

d) Zur Erklärung der Abhängigkeit der Zwillingsbildung von der Substratorientierung und der Substrattemperatur kann man das Modell von Holloway et al. ${ }^{6}$ anwenden. Danach werden um so höhere Substrattemperaturen zur Elimination der Zwillingsbildungen erforderlich sein, je kleiner der Winkel zwischen der Wachstums- und der jeweiligen $\langle 111\rangle$-Richtung ist. Da der kleinste Winkel zwischen einer $\langle 100\rangle$ - und einer $\langle 111\rangle$-Richtung $54,7^{\circ}$, zwischen einer $\langle 111\rangle$ - und einer $\langle 111\rangle$-Richtung $0^{\circ}$, zwischen einer $\langle 311\rangle$ - und einer $\langle 111\rangle$-Richtung $29,5^{\circ}$ beträgt, ergibt sich folgende

5 E. K. Müller, J. Appl. Phys. 35, 580 [1964].

${ }^{6}$ H. Holloway, K. Wollmann u. A. S. Joseph, Phil. Mag. 11, 263 [1965].

7 D. W. Pashley u. M. J. Stowell, Phil. Mag. 8, 1605 [1963]. 